\title{
GRAY-SCALE HOUGH TRANSFORM FOR THICK LINE DETECTION IN GRAY-SCALE IMAGES*
}

\author{
RONG-CHIN LO $\dagger$ and WEN-HSIANG TSAI $\ddagger$ \\ Department of Computer and Information Science, National Chiao Tung University, \\ Hsinchu, Taiwan 300, R.O.C
}

(Received 10 January 1994; in revised from 9 September 1994; received for publication 23 September 1994)

\begin{abstract}
A new extension of the Hough transform, called gray-scale Hough transform (GSHT), is proposed for detecting thick lines (called bands) in a gray-scale image. The use of the conventional Hough transform (CHT) usually requires the preprocessing steps of thresholding and edge detection (or thinning) before the transform can be performed on a gray-scale image containing bands. This causes loss of useful gray and position relationship existing among the pixels of a band, and requires certain postprocessing steps to recover the band in the original image. The proposed GSHT with a gray-scale image as the direct input removes this shortcoming, requiring neither preprocessing nor postprocessing step in detecting bands in a gray-scale image. Experimental results, showing the feasibility of the proposed approach, are also included.
\end{abstract}

$\begin{array}{lllll}\text { Gray-scale Hough transform } & \text { Gray-scale image } & \text { Thick lines } & \text { Band } & \text { Thresholding } \\ \text { Edge detection } & \text { Complexity } & \text { Radon transform }\end{array}$

\section{INTRODUCTION}

The conventional Hough transform $(\mathrm{CHT})^{(1,2)}$ is a well-known method for detecting and locating straight lines or analytic curves in images. It transforms a binary image into a Hough parameter counting space (HPCS). An HPCS consists of an array of cells, called accumultors, which are functions of the parameters of straight lines or analytic curves. In the CHT process, collinear points or co-curvilinear points that have the same set of parameters map to the same accumulator and each point increments the corresponding accumulator value by one to form a local maximum value in the HPCS. By way of detecting each local maximum value from the HPCS, a set of corresponding parameters can be found. A straight line or analytic curve can be detected and located with the set of parameters. A main advantage of the transform is its robustness to noise and discontinuities in images. In spite of this advantage it requires the preprocessing steps of thresholding and edge detection (or thinning) for an input image to become a point pattern with lines or curves of 1-pixel width. However, in many common images like Fig. 1, there exist gray-scale shapes consisting of band-type lines, arcs circles, ellipses, etc. Here, a line or curve segment is said to be of the band-type if the width of the segment is larger than 1 pixel. A

* This work was partially supported by National Science Council, Republic of China under contract number NSC800404-E009-19.

$\dagger$ Is also with the Department of Electronic Engineering, National Taipei Institute of Technology, Taipei, Taiwan 106, Republic of China.

$\ddagger$ To whom all correspondence should be addressed. gray-scale shape consisting of band-type line or curve segments will be called a gray-scale band-type shape or simply a gray band shape. As a result of performing the preprocessing steps before using the CHT, most gray-scale information in an input image is lost during thresholding and a lot of relationship among pixels is lost during edge detection (or thinning). In order to recognize the original gray band shape after the CHT, a priori knowledge of knowing where the band will appear or the use of a certain costly region connection operation is required. The CHT is so not suitable for direct use to detect band shapes in gray-scale images. This can be seen in many research works using the $\mathrm{CHT}^{(3-7)}$

To overcome these shortages of the CHT, a new extension of the CHT for gray band shape detection is proposed. Both the width and gray information of a shape are considered. This new extension will be called the gray-scale Hough transform (GSHT). The GSHT transforms an input gray-scale image directly into the HPCS, and includes an additional parameters, namely, the gray level. No image thresholding or thinning (or edge detection) is required. The new parameter space will be called the gray Hough parameter counting space (GHPCS). After performing the GSHT, the widths and gray values of bands are found by a band detection process using the resulting content of the GHPCS. For simplification while not losing generality, we focus discussions on detection of gray linear bands. Due to the use of the gray-level parameter, a gray linear band shape can be considered as a set of parallel connective straight lines with identical orientations and identical gray levels. All the pixels of one straight line map to an identical accumulator to form a local maximum 


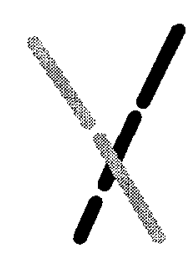

(a)

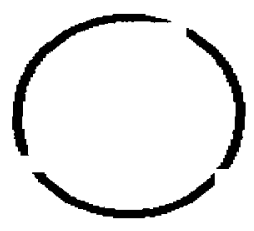

(b)

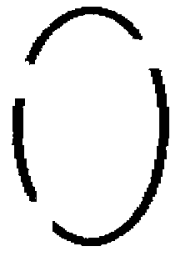

(c)

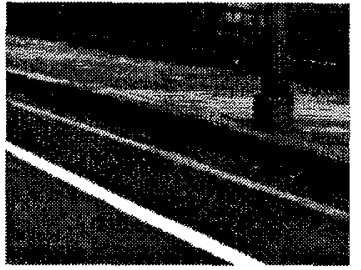

(d)

Fig. 1. Several kinds of band shapes existing in images. (a) Two gray linear band shapes. (b) A circular band shape. (c) An elliptical band shape. (d) An outdoor scene including two gray band-type path lines.

value in the HPCS, and the set of all the parallel connective straight lines map to a cluster of connected accumulators to form a local maximum plateau in the GHPCS

On the other hand, four features may be used to describe a gray linear band existing in a gray-scale image: the gray level, the orientation, the total number of pixels, and the length of the band. The value of these features can be calculated from the local maximum plateau to detect the gray linear band in the original image. The details are described in Section 3. Although the memory space complexity is 4-dimensional (4D), the order of time complexity is the same as that of the CHT. Nowadays the cost of memory is inexpensive, so the time of image processing is the primary consideration.

This paper is organized as follows. In Section 2 the CHT is first reviewed. In Section 3 the proposed GSHT and the use of the resulting GHPCS to detect bands in a gray-scale image are described in detail. Experimental results and discussions are given in Section 4. Some concluding remarks are given in Section 5.

\section{REVIEW OF CONVENTIONAL HOUGH TRANSFORM}

The CHT was originally described by Hough. ${ }^{(1)}$ Duda and Hart ${ }^{(2)}$ used it to detect straight lines in pictures. It also has received a number of improvements. ${ }^{(8-14)}$

The idea of using the CHT to detect a straight line can be illustrated by considering a set of collinear points forming a straight line in a binary image. Two parameters which may be used to describe a straight line are the distance $\rho$ and the orientation $\theta$ of the normal vector to the line from the image origin. ${ }^{(2)}$ Accordingly, a straight line can be defined by a relation $f$ with the following normal form:

$$
f((\rho, \theta),(x, y))=\rho-x \cos \theta-y \sin \theta=0 .
$$

From equation (1), it is easy to show that each point $\left(x_{i}, y_{i}\right)$ in the image coordinate system is mapped to a sinusoidal series of accumulators $A\left(\rho_{i}, \theta_{i}\right)$ in the HPCS For each pixel $\left(x_{i}, y_{i}\right)$ satisfying equation (1), the content of each of all the mapped accumulators is incremented by one. If the input image includes a straight line, a local maximum value in one of the accumulators can be detected from the HPCS. Accordingly, a pair of parameters, $\left(\rho_{i}, \theta_{i}\right)$, corresponding to the local maximum value can be found from the HPCS. By using the pair of parameters, the desired straight line can be detected in the input image.

Extension of the method to detect other curves (e.g., circles and ellipses) are straightforward. Although the $\mathrm{CHT}$ is a powerful method for detecting the presence of collinear or co-curvilinear points in binary images, it is not suitable to use it directly to detect band shapes in gray-scale images.

\section{PROPOSED GRAY-SCALE HOUGH TRANSFORM FOR DETECTING BANDS IN GRAY-SCALE IMAGES}

In this section, the proposed GSHT for band detection in gray-scale images is described in detail. For simplification, the discussions are focused on linear band shapes. For the sake of clarity, detection of linear bands in binary images using the CHT without performing the preprocessing steps of edge detection or thinning is treated first. Detection of linear bands in gray-scale images using the GSHT is discussed next. Finally, existence of a cluster of connected accumulators forming a local maximum plateau corresponding to a linear band is proved.

\subsection{Detection of linear bands in binary images using $C H T$}

Conventional Hough transform without edge detection or thinning. In conventional use of the Hough transform to detect a straight line, it needs to perform thresholding and edge detection (or thinning) in advance, and so a lot of gray and position information among pixels is lost. In this study, it is proposed to use the CHT, without performing the preprocessing steps of edge detection or thinning, to detect linear bands in binary images. The algorithm is as follows.

Algorithm 1. Conventional Hough transform without edge detection or thinning.

Input. A binary image containing white linear bands on black background, on which no edge detection or thinning has been performed.

Output. The values of an array of accumulators $A(\rho, \theta)$, in the HPCS. 
Steps.

(1) Initialize each accumulator content $A\left(\rho_{j}, \theta_{j}\right)$ to 0 .

(2) For each white pixel $\left(x_{i}, y_{i}\right)$,

(3) for $\theta_{j}=0^{\circ}$ to $179^{\circ}$ with increment of $1^{\circ}$,

(4) set $\rho_{j}=x_{i} \cos \left(\theta_{j}\right)+y_{i} \sin \left(\theta_{j}\right)$, and

(5) set accumulator value $A\left(\rho_{j}, \theta_{j}\right)=A\left(\rho_{j}, \theta_{j}\right)+1$.

(6) End.

Algorithm 1 directly maps the input binary image into the HPCS without edge detection or thinning. Therefore, the HPCS has more information than that obtained from the process of edge detection or thinning followed by the CHT. To detect a straight line, the two methods are the same, but to detect a linear band, the former is more suitable than the latter. Take Fig. 2 as an illustration. For each pixel $\left(x_{i}, y_{i}\right)$ in the image of Fig. 2(a), Algorithm 1 adds 1 to all mapped accumulators that form a sinusoidal curve in the HPCS. All collinear pixels forming a straight line in the original image have a common pair of parameters, $\left(\rho_{j}, \theta_{j}\right)$, which represents the normal vector of the straight line, and each of these pixels increments by one the value of an identical accumulator $A\left(\rho_{j}, \theta_{j}\right)$ located at the intersection point of a set of sinusoidal curves as shown in Fig. 2(b). The final value of $A\left(\rho_{j}, \theta_{j}\right)$ is equal to the total number of pixels on the corresponding straight line and represents the length of the straight line (assuming that the line has no gap and that the line length is measured by the number of line points). It forms a local maximum peak in the HPCS as shown in Fig. 2(c). From the viewpoint of geometric structures, a linear band is composed of many connective straight lines which are parallel to the two longer boundaries of the band, with identical orientations $\theta$, of equal lengths $L$, and with successively varying distances $\rho_{i}$, as shown in Fig. 2(d). After the CHT is performed, each of the parallel straight lines maps to a local maximum value, and all of the straight lines map to a row of successive local maximum values as shown in Fig. 2(e). So, the sum of all the values of the row of accumulators is equal to the total number of pixels in the band. On the other hand, each other line within the linear band, which is not parallel to the two longer band boundaries and is not a diagonal line in the band, is shorter and contains fewer pixels, and the value of its corresponding accumulator is smaller. Therefore, all the lines contained in the band map to a cluster of accumulators that form a local maximum plateau. For exmaple, for the band shown in Fig. 2(f), the local maximum plateau is shown in Fig. 2(g) or 2(h).

Detecting linear bands in binary image using the HPCS. For each linear band, a local maximum plateau exists in the HPCS, and it is found in this study that the linear band can be detected from the information of the accumulators in the local maximum plateau. Each linear band existing in the image is assumed to be with width greater than one pixel and length greater than a certain threshold value $t$. The local maximum plateau of each linear band is detected by discarding the accumulators whose values are less than the threshold value $t$. The thresholded local maximum plateau of each linear band forms a region in the HPCS. For the example of Fig. 2(g), three thresholded plateaus with different threshold values are shown in Figs 2(i) through $2(\mathrm{k})$.

Now, to detect the linear band from the information of the accumulators, three paramters of the band can be utilized: the orientation $\theta$, the total number of pixels and the length, of the linear band. With a fixed $\theta$ value, the last two parameters can be used to check possible band candidates. After detecting a plateau region in the HPCS as shown in any of Figs 2(i) through 2(k), each row of successive local accumulators along a certain value of $\rho$ at each orientation $\theta$ in the plateau region corresponds to a possible band candidate. As discussed previously, if $\theta$ is parallel to the orientation of the original linear band, the sum of the values of the row of the successive accumulators $A(\rho, \theta)$ is equal to the total number of pixels of the original linear band, and the average accumulator value [the sum divided by the total number of $A(\rho, \theta)$ ] is equal to the length of the band. Both the sum and the average values are maximum here. Under such two conditions, the value of $\theta$ is the optimal choice. These observations are illustrated in Figs 3(a) through to (d). Fig. 3(a) shows a linear band in an image with length $L_{0}$, orientation $\theta_{0}$, width $W_{0}$ and pixel number $N_{0}$. For Fig. 3(b), both of the two maximum conditions are true. The value $\theta_{1}$ is the optimal choice that can be used to retrieve the length and width of the linear band. For Fig. 3(c) or (d), the two maximum conditions are not met simultaneously. So, the values of $\theta_{2}$ and $\theta_{3}$ are not optimal choices. The steps for detecting linear bands in a binary image using the HPCS are described in the following.

Algorithm 2. Detecting linear bands in a binary image using the HPCS.

Input. A binary image including linear bands and a threshold value $t$.

Output. The parameters of all linear bands.

Steps.

(1) Perform the CHT of Algorithm 1.

(2) Detect all the local maxima in the HPCS whose values are greater than $t$ by thresholding the accumulator values $A(\rho, \theta)$ with threshold value $t$.

(3) Perform region growing ${ }^{(15)}$ to find all plateau regions.

(4) Check all labeled regions. In each region, for each $\theta_{i}$, compute the sum of all the accumulator values $A\left(\rho, \theta_{i}\right)$ and the average value for all $\rho$ in the region.

(5) For each region, search the local maximum value (denoted as $m$ ) of the average values computed in Step 4 , and find all possible band candidates by picking up in the neighborhood of the local maximum those average values which are larger than a certain fraction of $m$.

(6) Find the orientation $\theta_{m}$ whose sum value (computed Step 4) is locally maximum for all the candidates found in Step 5. 


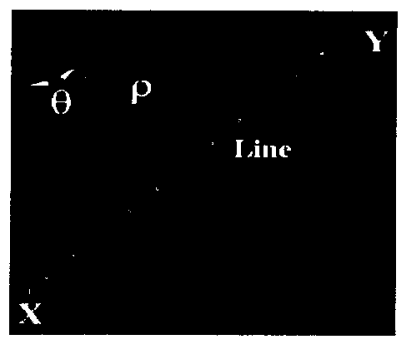

(a)

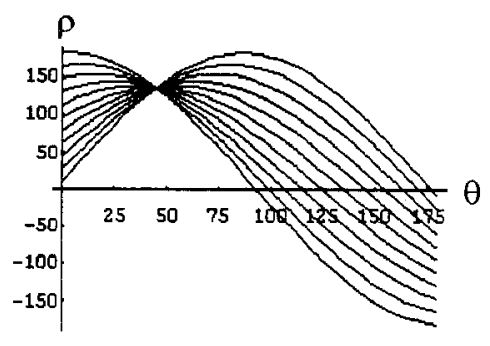

(b)

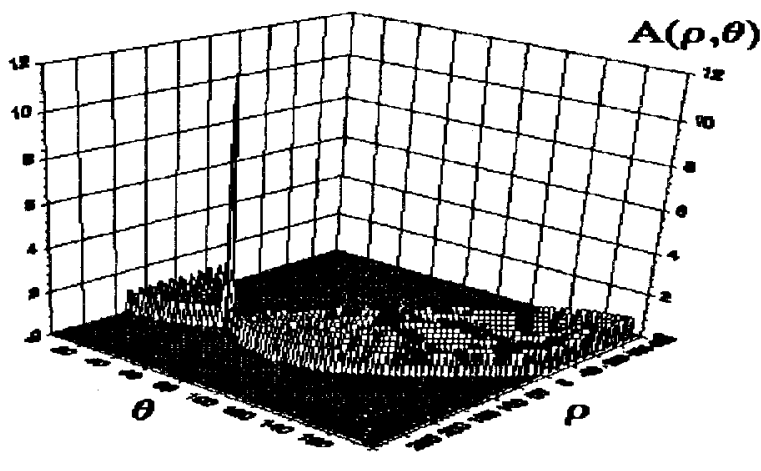

(c)

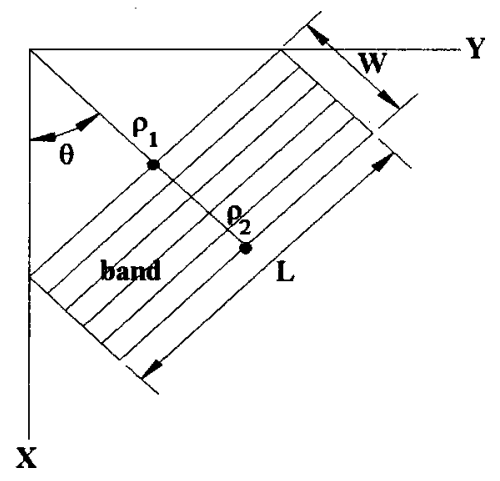

(d)

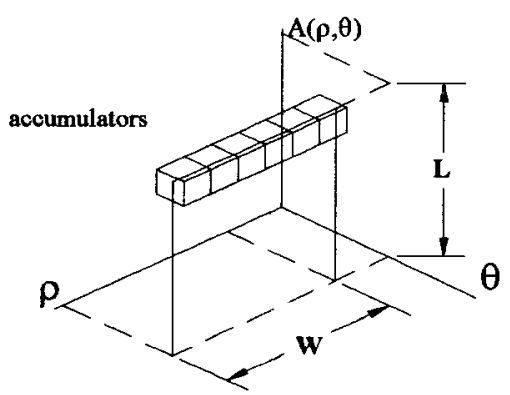

(e)

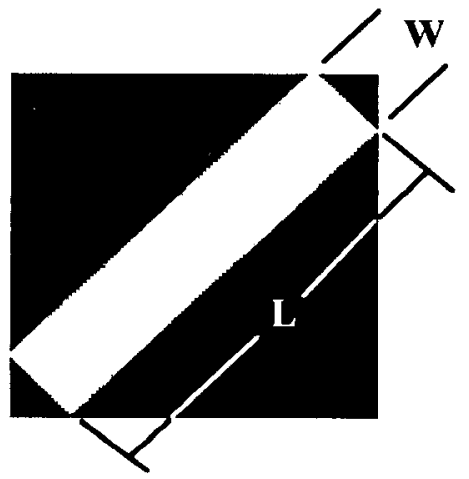

(f)

Fig. 2. Illustration of using CHT to detect a linear band in a binary image. (a) A set of collinear pixels with orientation $\theta=45^{\circ}$ in $\mathrm{x}-\mathrm{y}$ space. (b) The $2 \mathrm{D} \rho-\theta$ space after the CHT is applied. (c) A peak exists in the 3D HPCS $A(\rho, \theta)$ after the CHT is performed. (d) A linear band with length L and width W. (e) After the CHT is performed without edge detection or thinning, each of the parallel straight lines of (d) maps to a local maximum value in accumulators, and all the parallel straight lines map to a row of connective accumulators with local maximum values. (f) A linear band with slope of $45^{\circ}$. (g) A local maximum plateau (with height $\geq \mathrm{L}$ ) exists in the 3D HPCS after the CHT is performed on (f). (h) Same as (g) but with the HPCS viewed as an image. (i) The thresholded local maximum plateau with threshold value 145 . (j) The thresholded local maximum plateau with threshold value 230. (k) The thresholded local maximum plateau with threshold value 350 which is the same as illustrated in (e). 


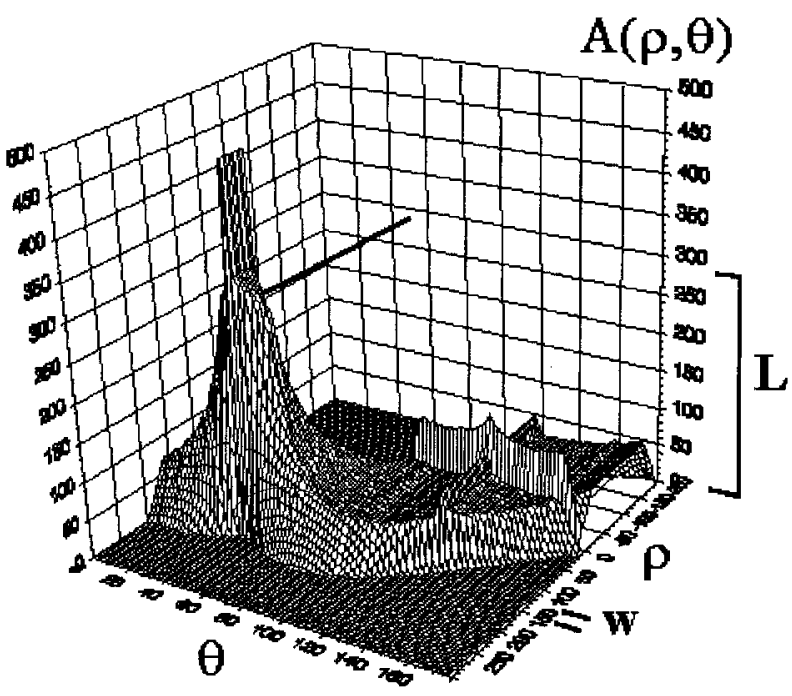

(g)

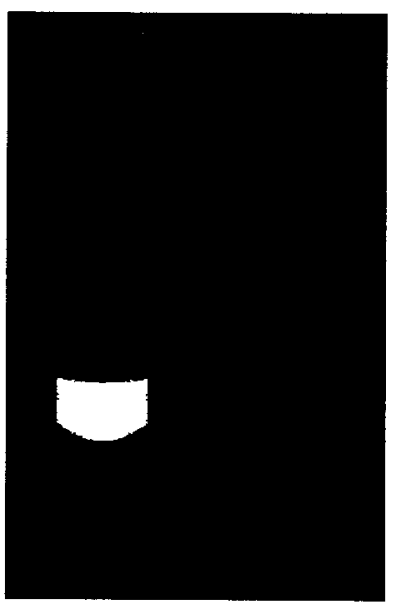

(i)

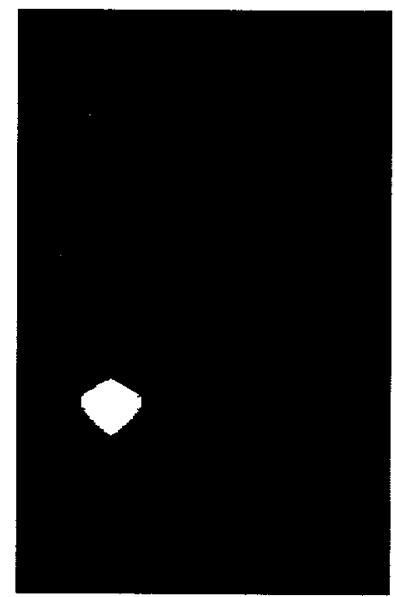

(j)

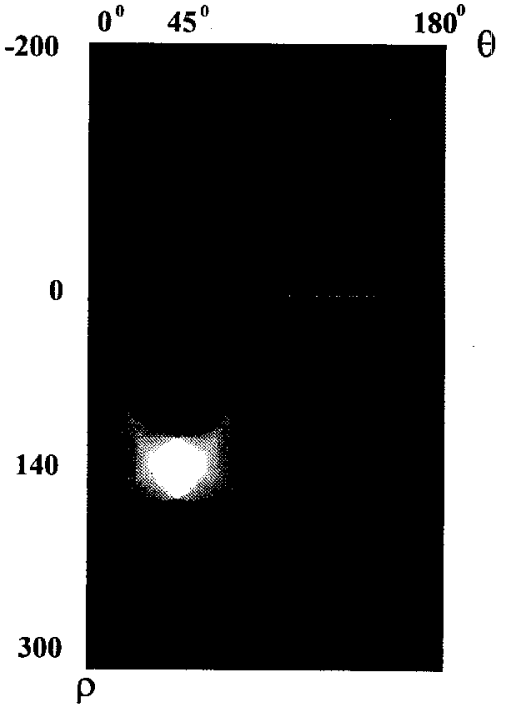

(h)

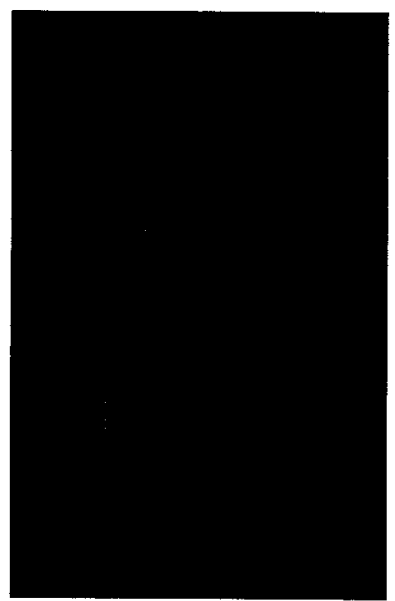

(k)

Fig. 2 (Cont.)

(7) Detect the two endpoints $\left(\rho_{1}, \theta_{m}\right)$ and $\left(\rho_{2}, \theta_{m}\right)$ of the region along $\rho$ at $\theta_{m}$.

(8) Output $\left|\rho_{1}-\rho_{2}\right|, \theta_{m}$, and $\left(\rho_{1}, \theta_{m}\right)$ and $\left(\rho_{2}, \theta_{m}\right)$ as the width, orientation and location of the linear band.

(9) End.

\subsection{Extension of CHT to GSHTfor detecting gray linear bands}

Gray-scale Hough transform (GSHT). It is easy to extend the CHT to the GSHT. The proposed GSHT directly maps a gray-scale image including linear bands [see Fig. 4(a) for an example] in the image coordinate space $(x, y, G)$ to the gray Hough parameter counting space (GHPCS) $A(\rho, \theta, G)$ without performing thres- holding and edge detection (or thinning) to the image in advance. Each pixel $\left(x_{i}, y_{i}\right)$ in the image coordinate space $(x, y)$ has gray value $G_{i}$, and each accumulator $A\left(\rho_{j}, \theta_{j}, G_{i}\right)$ in the GHPCS is a function of three parameters $\rho, \theta$ and $G$, where the definitions of $\rho$ and $\theta$ are the same as those of the CHT and $G$ represents the gray value. The new mapping relation $f$ is $f:(x, y, G) \rightarrow$ $A(\rho, \theta, G)$ with normal form:

$$
\begin{aligned}
f((\rho, \theta, G),(x, y, G))= & \rho(G)-x \cos (\theta(G)) \\
& -y \sin (\theta(G)) \\
= & 0 .
\end{aligned}
$$

The GHPCS is a 4D space as illustrated by Fig. 4(b), (c) or (d). The algorithm to compute the values of the accumulators $A\left(\rho_{j}, \theta_{j}, G_{i}\right)$ is as follows. 


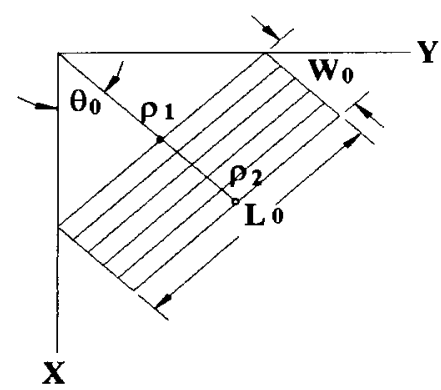

(a)

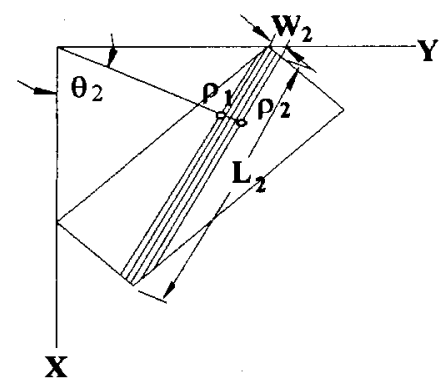

(c)

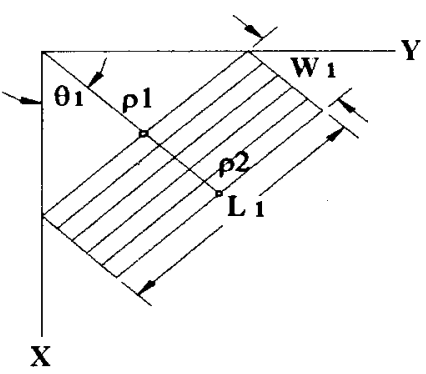

(b)

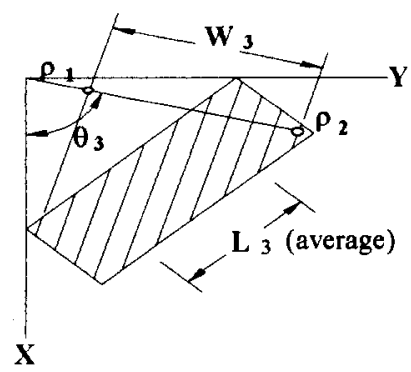

(d)

Fig. 3. Illustration of detecting a linear band in a binary image. (a) The original linear band with orientation $\theta_{0}$, length $L_{0}$, width $W_{0}$, and total number of pixels $N_{0}\left(=L_{0} W_{0}\right)$. (b) Both the maximum conditions, i.e., $L_{1}$ and $N_{1}\left(=L_{1} W_{1}\right)$, are satisfied. $\theta_{1}$ Is thus a good candidate of the $\theta_{0}$. (c) $L_{2} \approx L_{0}$ is well matched, but $N_{0}>N_{2}$

$\left(=L_{2} W_{2}\right)$, is not matched. (d) $N_{3}\left(=L_{3} W_{3}\right)$ matches well with $N_{0}$, but $L_{3}$ does not match with $L_{0}$.

Algorithm 3. Gray-scale Hough transform.

Input. A gray-scale image containing linear bands on which no thresholding and edge detection (or thinning) has been performed.

Output. The values of an array of accumulators $A(\rho, \theta, G)$ in the GHPCS.

Steps.

(1) Initialize each accumulator $A(\rho, \theta, G)$ to 0 .

(2) For each image pixel $\left(x_{i}, y_{i}, G_{i}\right)$,

(3) for $\theta_{j}=0^{\circ}$ to $179^{\circ}$ with increment of $1^{\circ}$,

(4) set $\rho_{j}\left(G_{i}\right)=x_{i} \cos \left(\theta_{j}\left(G_{i}\right)\right)+y_{i} \sin \left(\theta_{j}\left(G_{i}\right)\right)$, and

(5) set accumulator value $A\left(\rho_{j}, \theta_{j}, G_{i}\right)=$ $A\left(\rho_{j}, \theta_{j}, G_{i}\right)+1$.

(6) End.

For each line passing through a pixel $\left(x_{i}, y_{i}, G_{i}\right)$ satisfying equation (2), Algorithm 3 increments the value of each mapped accumulator $A\left(\rho_{j}, \theta_{j}, G_{i}\right)$ by one. Each set of collinear pixels with identical gray values, called a gray straight line, has three common parameters $(\rho, \theta, G)$ that represent the normal vector of the gray straight line, and all pixels in the line increments the value of an identical accumulator $A(\rho, \theta, G)$ as shown in Fig. 4(b). The final value of $A(\rho, \theta, G)$ is equal to the total number of pixels (or the length) of the gray straight line. It forms a local maximum value in the
GHPCS. From the viewpoint of geometric structures, a gray linear band is composed of many connective gray straight lines which have identical gray values and are parallel to the two longer boundaries of the band. All the parallel gray straight lines map to a row of successive accumulators $A(\rho, \theta, G)$ that are functions of two identical parameters $(\theta, G)$ and a varying parameter $\rho$, and have local maximum values. Like the CHT, the sum of all the values of the row of accumulators is equal to the total number of pixels in the gray linear band. On the other hand, each other line contained in the band, which is not parallel to the two longer boundaries of the band and not a diagonal line, is shorter and contains fewer pixels. Therefore all the lines in the band map to a cluster of accumulators that form a local maximum plateau in the 4D GHPCS.

Detection of linear bands in gray-scale images using the GHPCS. To detect gray linear bands existing in a gray-scale image like those in Fig. 4(a), four parameters of the band can be utilized: the gray level $G$, the orientation $\theta$, the total number of pixels and the length of the band. With a fixed $\theta$ value and an identical gray level $G$, the last two parameters can be used to check possible band candidates. Because the value of each accumulator represents the length of a corresponding 


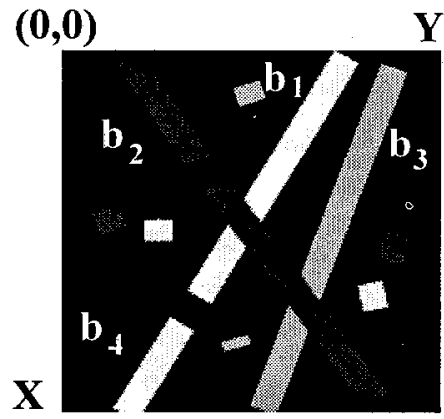

(a)

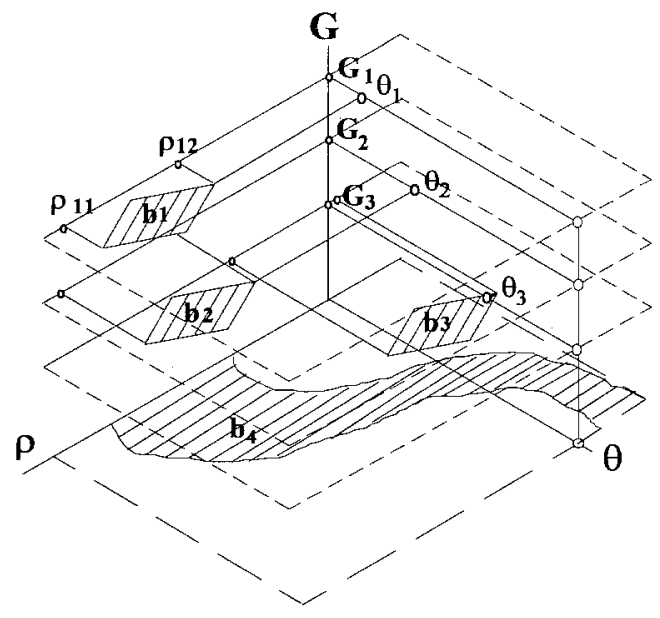

(c)

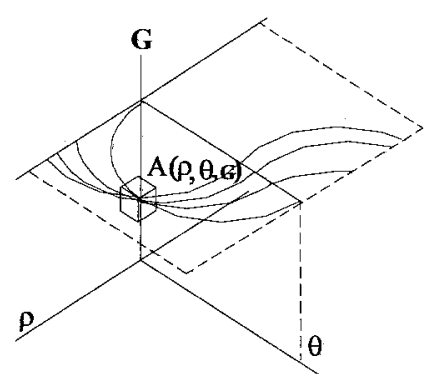

(b)

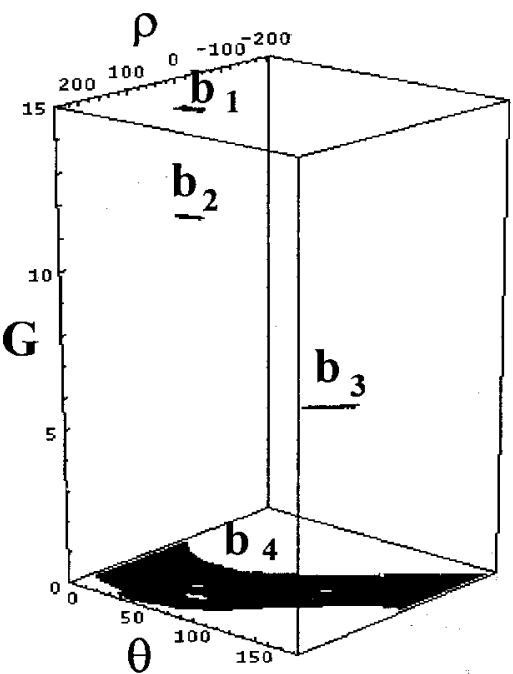

(d)

Fig. 4. Illustration of GSHT. (a) Three gray linear bands (denoted as $b_{1}, b_{2}$ and $b_{3}$ ) against a black background (denoted as $b_{4}$ ) existing in a gray-scale image with gray levels $0-15$. (b) An accumulator in the 4D GHPCS mapped to by a gray straight line. (c) Four plateau regions, filled by oblique lines and marked by $b_{1}, b_{2}, b_{3}$ and $b_{4}$, mapped to by the three gray linear bands and the background. (d) After the GSHT with threshold value $t=100$ is performed, four plateau regions can be found in the GHPCS, each on a gray level plane.

line, after the GSHT is performed, we discard accumulators whose values are less than a certain threshold value $t$ (i.e., we discard those lines whose lengths are smaller than a minimum acceptable value). A region growing process ${ }^{(15)}$ is performed to find all remaining thresholded connected regions in the GHPCS, called plateau regions, like those shown in Fig. 4(c) or (d). The sum of all the values of the accumulators $A(\rho, \theta, G)$ in each plateau region along the direction of $\rho$ at one certain orientation $\theta$ for one certain gray level $G$ is equal to the total number of pixels of a possible band candidate with gray level $G$. The length of the band candidate is equal to the sum divided by the total number of accumulators. When both the sum value and the length value are locally maximum simultaneously, the orientation $\theta_{m}$ and the gray level $G_{m}$ are the optimal choice of a gray linear band. For this plateau region, along $\rho$ at the values of $\left(\theta_{m}, G_{m}\right)$, we can find a row of connected accumulators each of whose values represents the length of a gray straight line parallel to the longer band boundaries and contained in the band, from which the width of the gray band can be detected (i.e., the width is equal to the total number of the row of the connected accumulators). The steps of the above gray band detection process are described in the following algorhthm.

Algorithm 4. Detecting linear bands in a gray-scale image.

Input. A gray-scale image including multiple gray linear bands and a threshold value $t$.

Output. The parameters of all gray linear bands. Steps.

(1) Perform the GSHT.

(2) Detect all the accumulators whose values are greater than threshold value $t$ by thresholding the accumulator values $A(\rho, \theta, G)$ with $t$.

(3) Perform region growing to find plateau regions. 
(4) Check all labeled plateau regions. In each region with gray level $G_{m}$, for each orientation $\theta$, compute the sum of all the accumulator values $A\left(\rho, \theta, G_{m}\right)$ and the average value along $\rho$ in the region.

(5) For each region, search the local maximum average value (denoted as $m$ ) of the average values computed in Step 4, and find all possible gray band candidates by picking up in the neighborhood of the local maximum those average values which are larger than a certain fraction of $m$.

(6) Find the orientation $\theta_{m}$ whose sum (computed in Step 4) is locally maximum for all the candidates found in Step 5.

(7) Detect the two endpoints $\left(\rho_{1}, \theta_{m}\right)$ and $\left(\rho_{2}, \theta_{m}\right)$ of the region along $\rho$ at $\left(\theta_{m}, G_{m}\right)$.

(8) Output $\left|\rho_{1}-\rho_{2}\right|, G_{m}, \theta_{m},\left(\rho_{1}, \theta_{m}\right)$ and $\left(\rho_{2}, \theta_{m}\right)$ as the width, the gray level, the orientation, and the location of the gray linear band.

(9) End.

\subsection{Mathematical meaning of the GSHT and detection of gray bands from the viewpoint of the Radon transform}

Anexpression for the CHT, as suggested by Deans ${ }^{(17)}$ and Leavers, ${ }^{(18)}$ may be written in a form of Radon transform ${ }^{(16)}$ as follows:

$$
\begin{aligned}
A(\rho, \theta) & =\mathscr{R}\{F(x, y)\} \\
& =\iint_{D} F(x, y) \delta(\rho-x \cos \theta-y \sin \theta) d x d y
\end{aligned}
$$

For the proposed GSHT, the form is

$$
\begin{aligned}
A(\rho, \theta, G) & =\mathscr{R}\{F(x, y, G)\} \\
& =\iint_{D} F(x, y, G) \delta(\rho-x \cos \theta-y \sin \theta) d x d y,
\end{aligned}
$$

where $A(\rho, \theta, G)$ is a density function which is equivalent to the accumulator array for gray value $G$ in the 4D GHPCS, and $F(x, y, G)$ represents a gray linear band in the input gray image $D$. Equation (3) is a special case of equation (4) with the gray value $G$ being constant. Also, in our case,

$$
F(x, y, G)= \begin{cases}1, & \text { in } D \\ 0, & \text { otherwise }\end{cases}
$$

The Dirac delta function $\delta$ forces an integration of $F(x, y, G)$ along a line whose normal form is $\rho-x \cos \theta-$ $y \sin \theta=0$.

Assume that all the linear gray bands have lengths greater than $t$. Also, recall that $A(\rho, \theta, G)$ is equal to the length of a gray line contained in and parallel to a gray linear band. Define $A_{t}(\rho, \theta, G)$ as follows:

$$
A_{t}(\rho, \theta, G)= \begin{cases}A(\rho, \theta, G), & \text { if } A(\rho, \theta, G) \geq t ; \\ 0, & \text { otherwise, }\end{cases}
$$

where $t$ is a threshold value. That is, $A_{t}(\rho, \theta, G)=$ $A(\rho, \theta, G) \Theta(A(\rho, \theta, G)-t)$ where the step function $\Theta$ is such that $\Theta(p)=1$ if $p \geq 0$, and $\Theta(p)=0$, otherwise. All the $A_{t}(\rho, \theta, G)$ values form a local maximum plateau for the gray linear band in the 4D GHPCS. Let
$N_{t}\left(\rho_{1}, \rho_{2}, \theta, G\right)$ represent the total number of pixels and $W_{f}\left(\rho_{1}, \rho_{2}, \theta, G\right)$ the average length, of the gray linear band, where $\left(\rho_{1}, \theta\right)$ and $\left(\rho_{2}, \theta\right)$ are the parameters of the two longer boundaries of the gray linear band. Then, for each local maximum plateau we have ${ }^{(16)}$

$$
\begin{aligned}
N_{t}\left(\rho_{1}, \rho_{2}, \theta, G\right) & =\int_{\rho_{1}}^{\rho_{2}} A_{t}(\rho, \theta, G) d \rho \\
& =\int_{\rho_{1}}^{\rho_{2}} A(\rho, \theta, G) \Theta(A(\rho, \theta, G)-t) d \rho, \\
W_{t}\left(\rho_{t}, \rho_{2}, \theta, G\right) & =N_{t}\left(\rho_{1}, \rho_{2}, \theta, G\right) /\left|\rho_{1}-\rho_{2}\right| .
\end{aligned}
$$

When both $N_{t}\left(\rho_{1}, \rho_{2}, \theta, G\right)$ and $W_{t}\left(\rho_{1}, \rho_{2}, \theta, G\right)$ are locally maximum simultaneously at an orientation $\theta_{m}$ for a certain gray level $G_{m}$, the orientation $\theta_{m}$ and the gray level $G_{m}$ are the optimal choice for the gray linear band, and $\left(\rho_{1}, \theta_{m}\right)$ and $\left(\rho_{2}, \theta_{m}\right)$ specify the two longer boundaries of the linear gray band.

\subsection{Proving existence of a local maximum plateau in the HPCS or the GHPCS for each linear band in an image}

After using the CHT to map a straight line to the HPCS, a local maximum peak is formed in the HPCS. The result of mapping a linear band with the CHT of Algorithm 1 or the GSHT of Algorithm 3 also has such a property except that it is a plateau in the HPCS or the GHPCS. This fact will be proved in this section. Without loss of the generality, only the case of using the CHT to detect a linear band with orientation $45^{\circ}$ like that known Fig. 5(a) is discussed. The case of using the GSHT can be similarly derived.

Assume that $L_{2}$ and $L_{3}$ are the two longer boundary lines of the linear band, and $L_{1}$ and $L_{4}$ are the two diagonal lines contained in the linear band, as shown in Fig. 5(b). Let their normal vectors be denoted as $\left(\rho_{1}, \theta_{i}\right)$ for $i=1,2,3$ and 4 . After the CHT of algorithm 1 is performed, it is obvious to see the following relations:

(1) $\rho_{2}<\rho_{1}<\rho_{3}, \rho_{2}<\rho_{4}<\rho_{3}$,

(2) $\theta_{1}<\theta_{2} \cong \theta_{3}<\theta_{4}$, and

(3) $L_{2} \leq L_{1} \cong L_{4} \leq L_{3}$.

Given an unknown linear band, it includes many possible linear bands. For each possible linear band with orientation $\theta_{i}$, its average length is defined as the average of all the straight lines $L_{j}$ with parameters $\left(\rho_{j}, \theta_{i}\right)$ contained in the band and parallel to the two longer boundary lines of the band. It is assumed that on each of the lines, the total number of pixels is more than a certain threshold value $t$ (i.e., each of the lines is longer than a minimum acceptable value). Let the average length of the band be denoted as $L_{a v}\left(\theta_{i}\right)$. The maximum of all the values of $L_{a v}\left(\theta_{i}\right)$ is the average length of the unknown linear band. Let it be denoted as $L_{a v}\left(\theta_{m}\right)$, where $\theta_{m}$ is the orientation of the unknown linear band. In our case of Fig. 5, $L_{a v}\left(\theta_{m}\right)$ is equal to $\left(L_{2}+L_{3}\right) / 2$ and $\theta_{m} \cong \theta_{2} \cong \theta_{3}$. From the above three relations, we discuss two cases: one is that a straight line is almost parallel to the unknown linear band, the 
other is on the contrary. In Case 1 , given a straight line $L_{i}$ with normal vector $\left(\rho_{i}, \theta_{i}\right)$ almost parallel to the unknown linear band, then $\theta_{i} \cong \theta_{m} \cong \theta_{2} \cong \theta_{3}$. From the above three relations, we have $\theta_{i}$ in $\left[\theta_{1}, \theta_{4}\right], \rho_{i}$ in $\left[\rho_{2}, \rho_{3}\right]$, and its length $L_{i}$ in $\left[L_{2}, L_{3}\right]$. Also, because the value of $A\left(\rho_{i}, \theta_{i}\right)$ is equal to the total number of pixels (or the length) of the straight line $L_{i}$ from relation (3) above, the following relations can be derived:

$A\left(\rho_{2}, \theta_{2}\right) \leq A\left(\rho_{i}, \theta_{i}\right) \cong A\left(\rho_{1}, \theta_{1}\right) \cong A\left(\rho_{4}, \theta_{4}\right) \leq A\left(\rho_{3}, \theta_{3}\right)$.

These facts mean that the accumulators $A\left(\rho_{i}, \theta_{i}\right)$ fall in a region bounded by the accumulators $A\left(\rho_{1}, \theta_{1}\right)$, $A\left(\rho_{2}, \theta_{2}\right), A\left(\rho_{3}, \theta_{3}\right)$ and $A\left(\rho_{4}, \theta_{4}\right)$ in the HPCS and the values of all the accumulators $A\left(\rho_{i}, \theta_{i}\right)$ are approximately equal and form a group in the HPCS, as shown in Fig. 5(c) or (d). In Case 2, given a line in the linear band considerably not parallel to the two longer boundaries of the band, i.e., with $\theta_{i}$ not in $\left[\theta_{1}, \theta_{4}\right]$, its length will be much smaller than that of the band. That is, the accumulator $A\left(\rho_{i}, \theta_{i}\right)$ has a value much smaller than those within the previously-mentioned region and is not surrounded by the region. As a summary of the two cases, we can conclude that in the HPCS, the mapping of the linear band forms a plateau as shown in Fig. 5(c) or (d). Figure 5(e) and (f) show the plateau region viewed as an image and from $3 \mathrm{D}$ viewpoint, respectively.

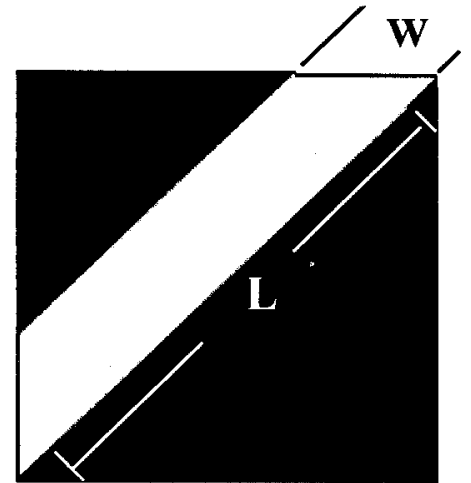

(a)

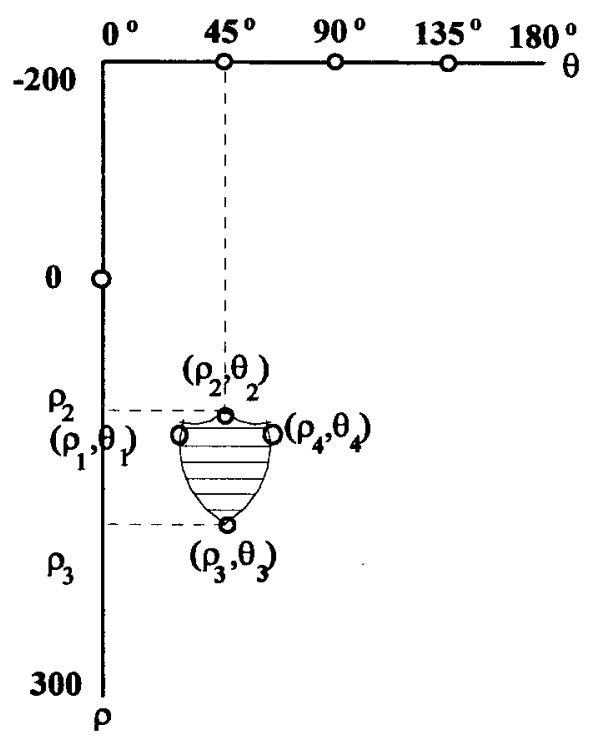

(c)

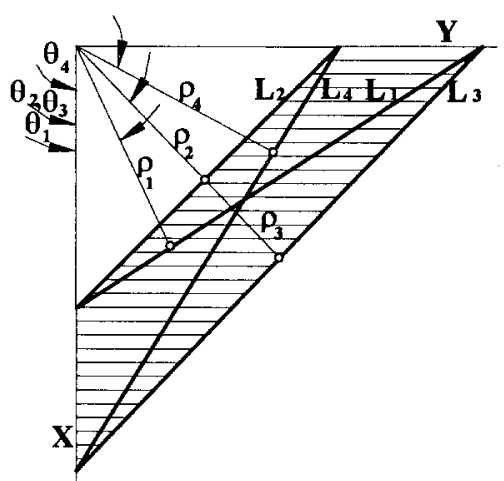

(b)

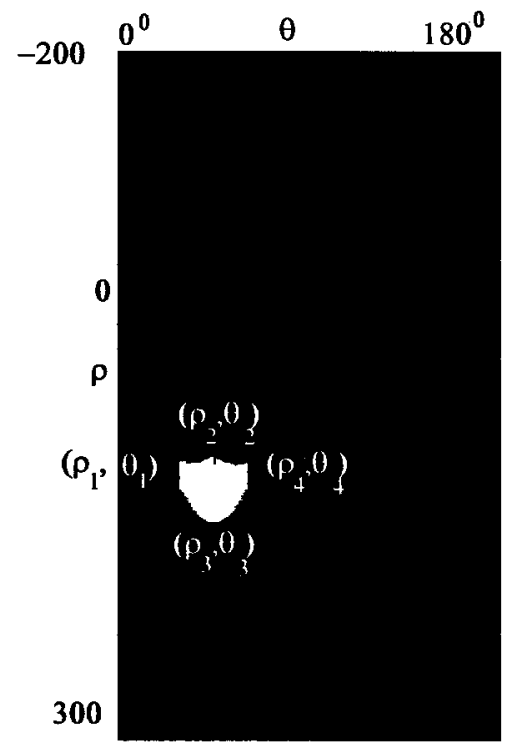

(d)

Fig. 5. Illustration of existence of a local maximum plateau in the HPCS for each linear band. (a) A linear band with orientation $45^{\circ}$. (b) $L_{i}\left(\rho_{i}, \theta_{i}\right)$ which are edges and diagonal lines of the band. (c) A cluster of accumulators, filled by oblique lines, with local maximum values which form a region. (d) After CHT, accumulator values larger than 225 forms a plateau region in the HPCS. (e) The HPCS viewed as an image.

(f) (e) viewed from $3 \mathrm{D}$ viewpoint. 


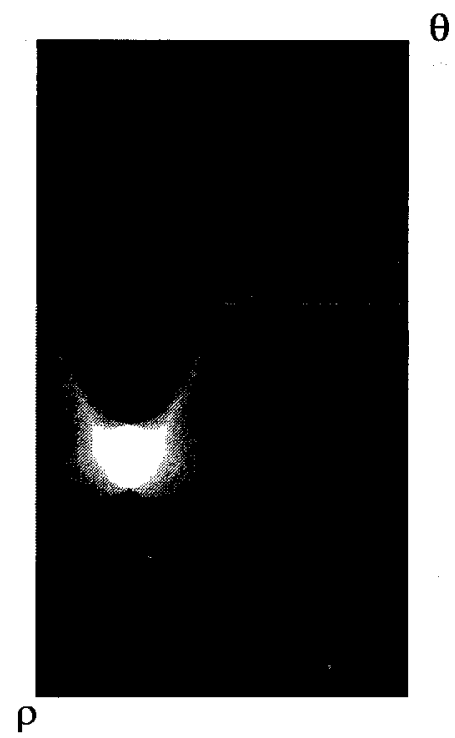

(e)

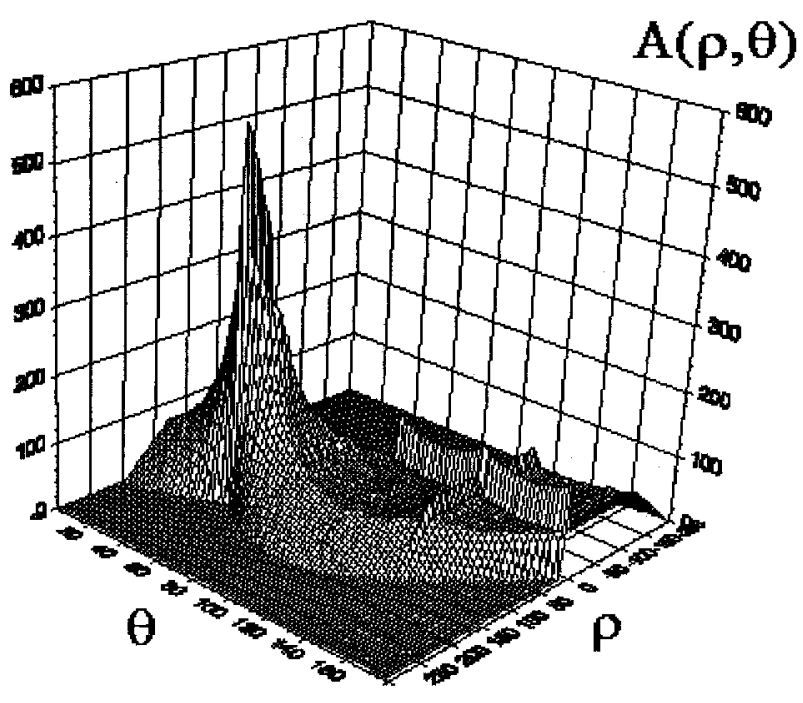

(f)

Fig. 5 (Cont.)

\section{EXPERIMENTAL RESULTS AND DISCLSSION}

\subsection{Experimental results}

The GSHT algorithm has been implemented on a SUN SPARC 10 workstation and a set of images has been tested. Due to the limitation of memory size, the gray values $(0-255)$ of each input image are requantized uniformly into 16 gray lévels (G0-G15) and the size of each image is limited to $200 \times 200$ pixels. Some results are shown in Fig. 6 through to Fig. 8. Use of the CHT to detect noisy and discontinuous linear bands in a binary image is shown in Fig. 6. Use of the GSHT to detect noisy and discontinuous linear bands in a grayscale image is shown in Fig. 7. An example of practical applications to real images is shown in Fig. 8. Figure 6(a) is the input image. Fig. 6(b) is the result of mapping into the HPCS viewed as an image including two local maximum plateaus (marked by a and b). Figure 6(c) shows the two detected bands. Figure $6(\mathrm{~d})$ is the same as Fig. 6(b) but viewed from 3D view point. Figure 7(a) is a gray-scale image including three linear bands with different gray values against a black background. Figure 7(b) shows all the detected bands (including the background, regarded as a band). Figure $7(\mathrm{c})$ is the same as Fig. 7(b) but with the background excluded. After the GSHT and the detection algorithm are performed, the output in the GHPCS containing four local maximum plateaus marked by a, b, c and $d$ is shown in Fig. 7(d). Figure 7(e) is the same as Fig. 7(d) except that the plateau of the background is removed. Figure $8(a)$ is the outdoor scene of Fig. 1(d) requantized uniformly with 16 gray levels. Figure 8 (b) shows all the detected bands. In actual applications, the prior knowledge about the range of band gray levels may be used.
Figure 8(c) shows the result of the detected bands with gray levels equal to 5 (the curb). Figure 8(d) shows the result of all the detected bands with gray levels greater than or equal to 10 (the path lines). Figure $8(\mathrm{e})$ shows the result of mapping into the 4D GHPCS. Figure 8(f) corresponds to Fig. 8(c) and Fig. 8(g) corresponds to Fig. 8(d). Note that the scales of both Fig. $8(\mathrm{f})$ and Fig. 8(g) have been magnified.

\subsection{Discussion}

Due to the memory limitation, all tested gray-scale images used in our experiments were requantized into 16 gray levels. However, this is not necessary; if the memory is sufficient, we may directly perform the GSHT on the gray-scale image without any preprocessing step. Furthermore, as seen from Algorithm 1 (the $\mathrm{CHT}$ without edge detection or thinning) and Algorithm 3 (the GSHT without any thresholding and quantization), the steps of the two algorithms are the same as those of the conventional CHT. Thus, they are almost the same in cost and complexity.

Furthermore, in Algorithm 4, all the local maxima $A(\rho, \theta, G)$ in the GHPCS are thresholded by $t$ and region growing are performed to form plateau regions. If two bands are almost parallel and very close to each other, and their gray-levels are almost the same, then the two regions corresponding to the two bands in the GHPCS will join together and the proposed method will brake. However, as long as the two bands have different distances $\rho$ and gray values $G$, the two regions can be separated and the two bands can be detected by our method.

Finally, because the length of a straight line is defined by the number of pixels in the line which appears 


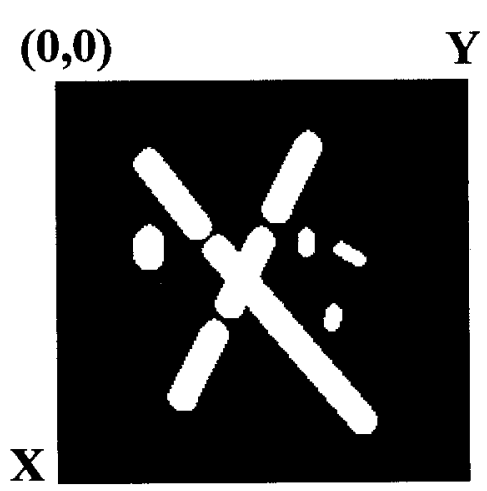

(a)

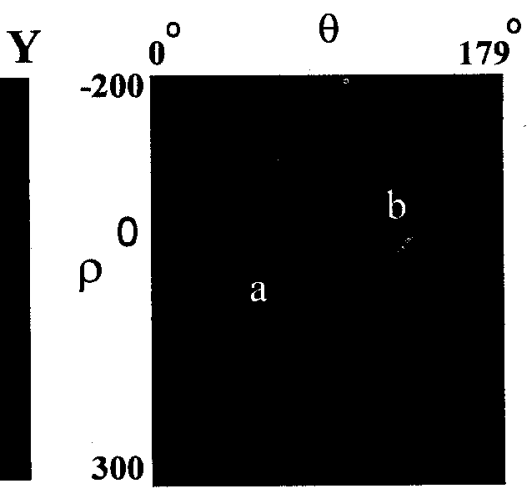

(b)

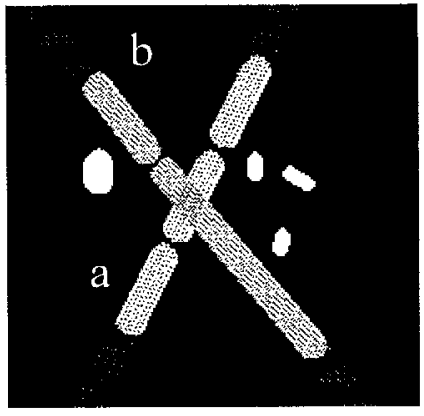

(c)

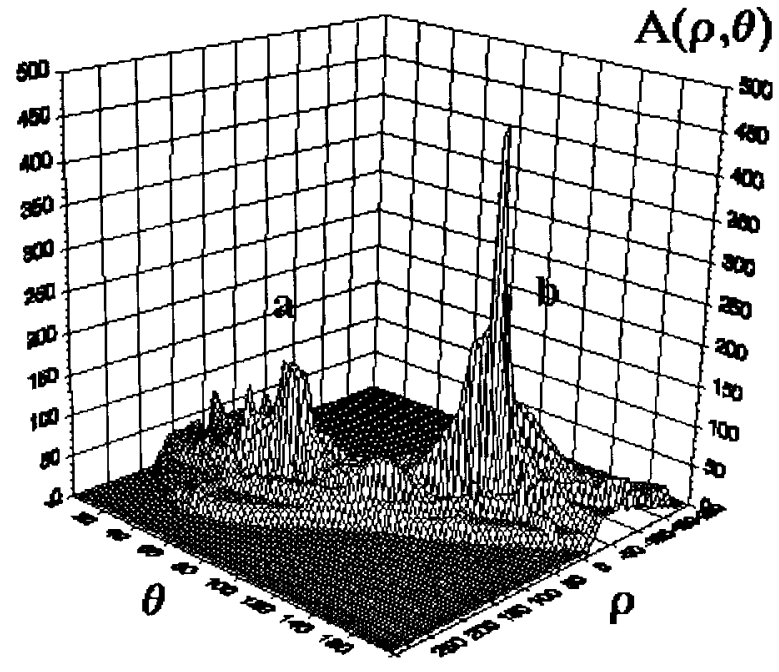

(d)

Fig. 6. Experimental results of linear band detection in a binary image. (a) Input image including two long binary linear bands. (b) Output image including two clusters of accumulators in th HPCS. (c) Two linear bands are detected with threshold value $t=120$. (d) (b) viewed from 3D viewpoint. 


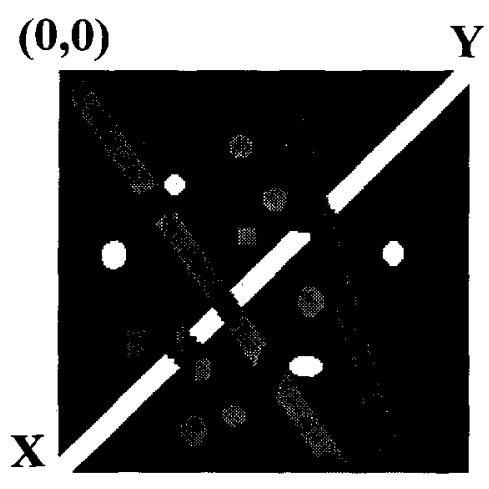

(a)

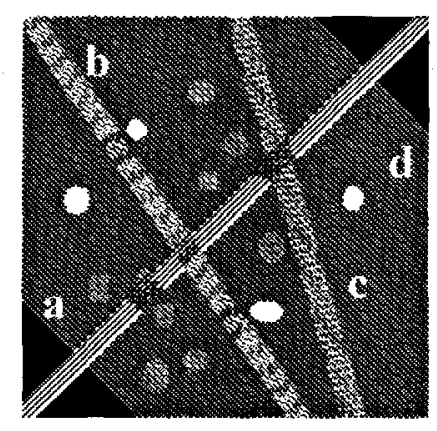

(b)

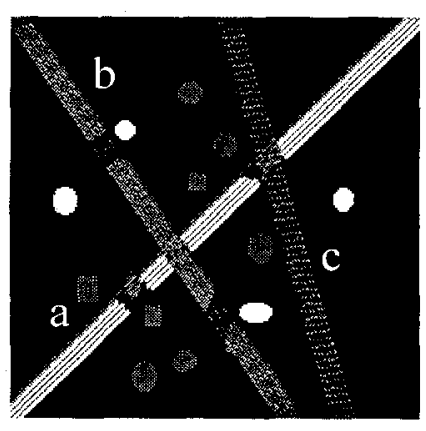

(c)

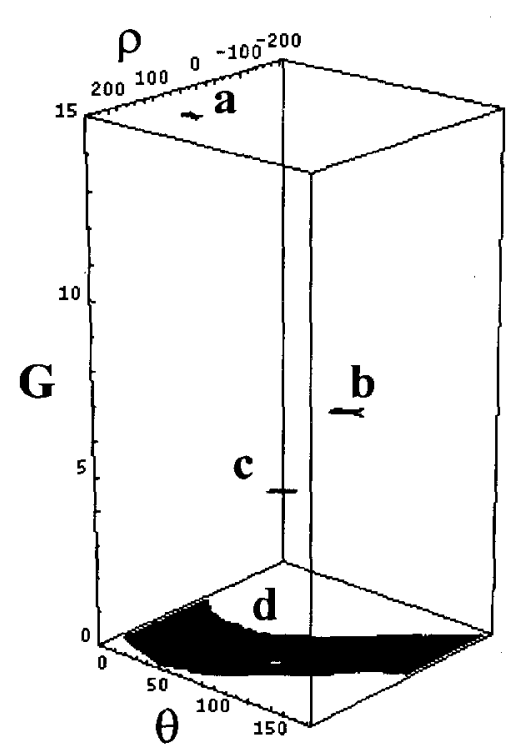

(d)

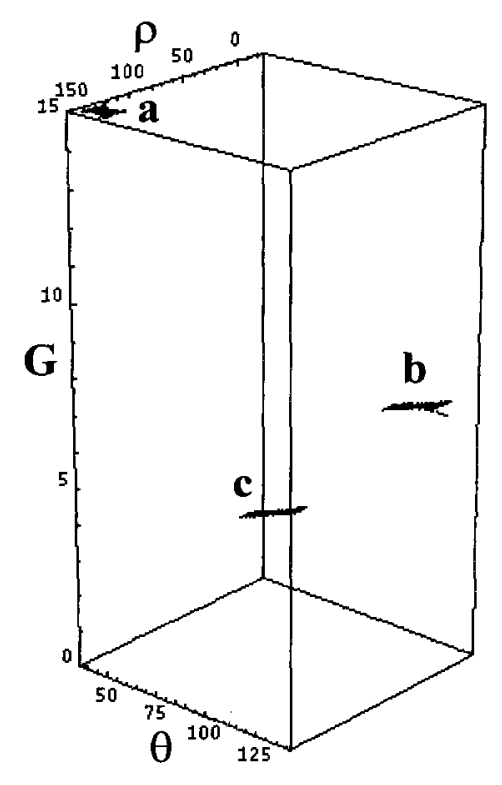

(e)

Fig. 7. Experimental results of gray linear band detection in a gray-scale image. (a) Input image including three gray linear bands and one background (also a band). The image only has 16 gray levels. (b) After GSHT with threshold value $t=100$, four gray bands, marked by $a, b, c$ and $d$ are detected. The one with mark $d$ is the background. (c) The same as (b) but with the "background band" removed. (d) The output of GSHT shown in the 4D GHPCS. The gray levels (G) of a, b, $c$ and $d$ are $15,7,5$ and 0 , respectively. (e) The same as (d) except that the background is discarded and that the scale is magnified a little. 


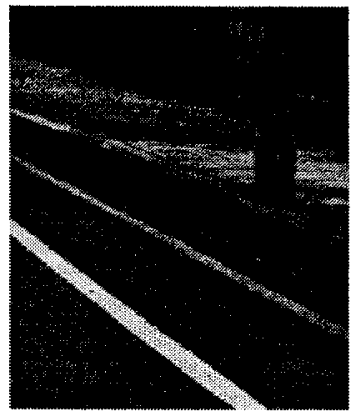

(a)

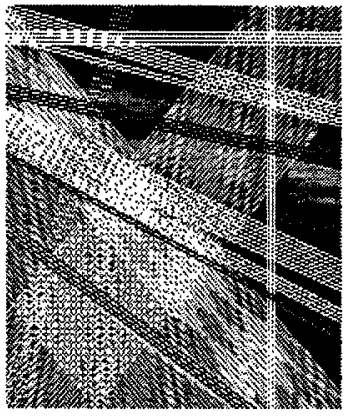

(b)

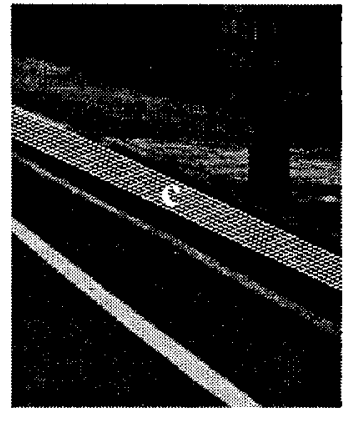

(c)

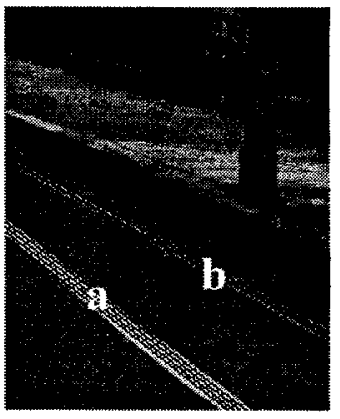

(d)

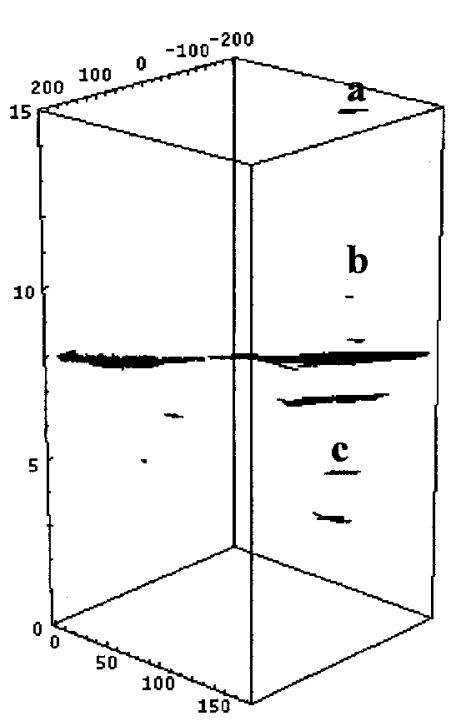

(e)

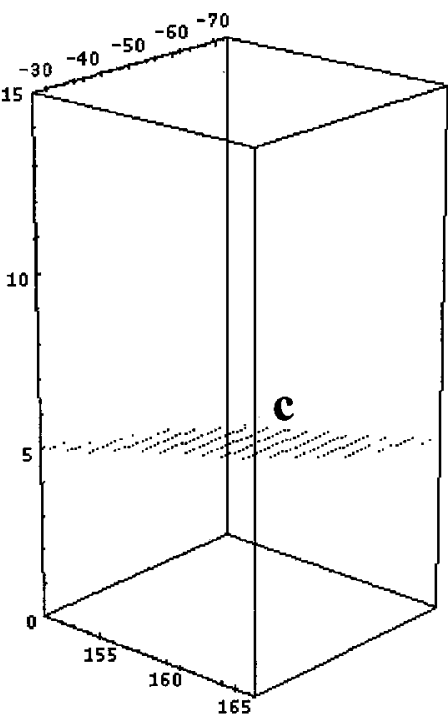

(f)

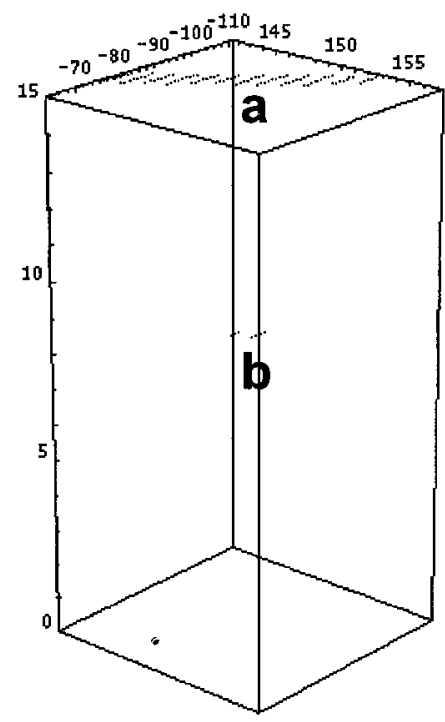

(g)

Fig. 8. Experimental results of gray linear band detection in an outdoor scene. (a) Input outdoor scene with 16 gray levels. (b) All the detected gray bands with threshold value $t=93$ superimposed on the image. (c) The same as (b) except that only the band gray level fixed at 5 is shown. The detected gray band is marked by c. (d) The same as (b) except that only the bands with gray levels greater than or equal to 10 are shown. The detected gray bands are marked by a and b, respectively. (e) The output of mapping into the 4D GHPCS. (f) The mapping into the GHPCS corresponding to (c). (g) The mapping into the GHPCS corresponding to (d). 


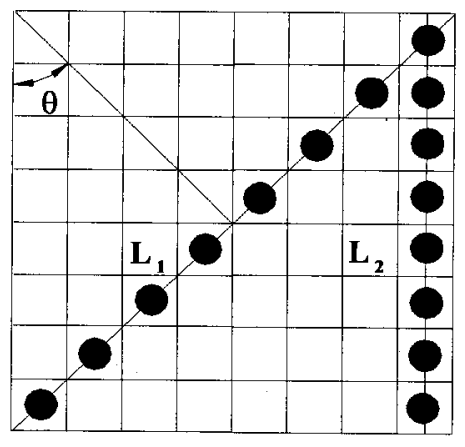

Fig. 9. Due to the definition of the length of a line in terms of the number of pixels in the line, the real length of $L_{1}$ is larger than the measured length.

finally as the value of an accumulator $A(\rho, \theta)$ after the CHT or the GSHT is performed, and due to the discretization of the Hough transform, errors of the measured lengths of lines in an image can be observed. An example is shown in Fig. 9 from which it can be seen that $L_{1}$ and $L_{2}$ with different lengths have the same number of pixels. In other words, their measured lengths are equal, but actually they are not of equal length. Therefore, certain correction of the final contents of the accumulator values must be made. It is found in this study that if we multiply the final contents of the accumulators values $A(\rho, \theta)$ by a factor $\mathrm{K}$ according to the value of $\theta$ in the following way, the resulting $A(\rho, \theta)$, and so the resulting corresponding line length, becomes more reasonable: (1) for $0^{\circ} \leq \theta<45^{\circ}, \mathrm{K}=$ $1 / \cos (\theta)$, (2) for $45^{\circ} \leq \theta<90^{\circ}, K=1 / \sin (\theta)$, (3) for $90^{\circ} \leq \theta<135^{\circ}, K=1 / \cos (\theta-90)$, and (4) for $135^{\circ} \leq$ $\theta<180^{\circ}, \mathrm{K}=1 / \sin (\theta-90)$. This correction is performed right after Algorithm 1 or Algorithm 3 is completed.

\section{CONCLUSIONS}

A new approach to gray linear band detection using a new extension of the CHT, namely the GSHT, without the need of the preprocessing steps of thresholding and edge detection (or thinning) has been proposed. The approach is robust to gray scales, noise and discontinuity in images. Although the space complexity is one dimension higher than the CHT, the time complexity is the same. The CHT can be viewed as a special case of the GSHT with input shapes being one-pixel wide with two gray levels. From the experimental results it was seen that the GSHT has high potential for applications. How to extend the GSHT to detect curvilinear bands is worth further study. It is also interesting to extend the GSHT to detect color bands.

\section{REFERENCES}

1. P. V. C. Hough, Method and Means for Recognizing Complex Patterns, U.S. Patent 306954 (1962).

2. R. O. Duda and P. E. Hart, Use the Hough transform to detect lines and curves in pictures, Comm. Assoc. Comput. 15, 11-15 (1972).

3. J. Illingworth and J. Kittler, A survey of the Hough transform, Comput. Vision, Graphics Image Process. 44, $87-116(1988)$.

4. S. N. Srihari and V. Govindaraju, Analysis of textual images using the Hough transform, Mach. Vision Applic. 2, 141-153 (1989).

5. W. Niblack and D. Petkovic, On improving the accuracy of the Hough transform, Mach. Vision Applic. 3, 87-106 (1990).

6. T. M. Vanveen and F. C. Groen, Discretization errors in the Hough transform, Pattern Recognition 14, 137-145 (1981).

7. V. F. Leavers, Which Hough transform? CVGIP: Image Understanding 58, 250-264 (1993).

8. M. Atiquzzaman, Multiresolution Hough transforman efficient method of detecting patterns in images, IEEE Trans. Patt. Anal. Mach. Intell. 14, 1090-1992 (1992).

9. N. Kiryati, Y. Eldar and A. M. Bruckstein, A probabilistic Hough transform, Pattern Recognition 24, 303-316 (1991).

10. H. W. Li, M. A. Lavin and R. J. LeMaster, Fast Hough transform: a hierarchical approach, Comput. Vision Graphics Image Process. 36, 139-161 (1986).

11. J. M. Jolion and A. Rosenfeld, An O( $\log n)$ pyramid Hough transform, Pattern Recognition Lett. 9, 343-349 (1989).

12. L. Xu and E. Oja, Randomized Hough transform (RHT): basic mechanisms algorithms, and computational complexities, CVGIP: Image Understanding 57, 131-154(1993).

13. T. W. Kao, S. J. Horng, Y. L. Wang and K. L. Chung, A constant time algorithm for computing Hough transform, Pattern Recognition 26, 277-286 (1993).

14. S. Y. K. Yuen, T. S. L. Lam and N. K. D. Leung, Connective Hough transform, Image Vision Comput. 11, 295-301 (1993).

15. A. Rosenfeld and A. C. Kak, Digital Picture Processing. Academic Press, New York (1982).

16. I. M. Gel'fand, M. I. Graev and N. Ya. Vilenkin, Generalized Function Vol 5, Integral Geometry and Representation Theory. Academic Press, New York (1966).

17. S. R. Deans, Hough transform from the Radon transform, IEEE Trans. Pattern Anal. Mach. Intell. 3, 185-188(1981).

18. V. F. Leavers, Use of the Radon transform as a method of extracting information about shape in two dimensions, Image Vision Comput. 10, 99-107 (1992).

\begin{abstract}
About the Author-RONG-CHIN LO was born in Miao-Li, Taiwan, Republic of China on December 25, 1950. He received the B.S. degree in the Department of Physics from National Cheng-Kung University in 1974 and the M.S. degree in the Department of Electrical Engineering from Tatung Institute of Technology in 1982. He worked as a Supervisor at Great Ltd from 1982 to 1984, and as a Deputy Manager at China Data Processing Center from 1984 to 1985 . The major work was to develop new products. He joined the Department of Electronic Engineering at St John's \& St Mary's Institute of Technology from 1985 to 1989 . In August 1989, he joined the Department of Electronic Engineering at National Taipei Institute of Technology, and is currently a Lecturer there. He is currently working toward his Ph.D. Degree in Computer and Information Science at National Chiao Tung University. His recent interests include pattern recognition, image processing and autonomous vehicle navigation.
\end{abstract}


About the Author - WEN-HSIANG TSAI was born in Tainan, Taiwan, Republic of China on 10 May, 1951. He received the B.S. degree from National Taiwan University, Taipei, Taiwan, Republic of China in 1973, the M.S. degree from Brown University, Providence, Rhode Island, U.S.A. in 1977, and his Ph.D. degree from Purdue University, West Lafayette, Indiana, U.S.A. in 1979, all in electrical engineering. Since November 1979, he has been on the faculty of the Institute of Computer Science and Information Engineering at National Chiao Tung University, Hsinchu, Taiwan. From 1984 to 1986, he was an Assistant Director and later an Associate Director of the Microelectronics and Information Science and Technology Research Center at National Chiao Tung University. He joined the Department of Computer and Information Science at National Chiao Tung University in August 1984, acted as the Head of the Department from 1984 to 1988 , and is currently a Professor there. He serves as a consultant to several research institutes and industrial companies. His current research interests include computer vision, image processing, pattern recognition, and Chinese information processing. Dr Tsai is an Associate Editor of Pattern Recognition, the Journal of the Chinese Institute of Engineers, and the Journal of Information Science and Engineering, and was an Associate Editor of the International Journal of Pattern Recognition and Artificial Intelligence, Computer Quarterly and the Proceedings of National Science Council of the Republic of China (Part A). He was elected as an Outstanding Talent of Information Science of the Republic of China in 1986. He was the winner of the 13th Annual Best Paper Award of the Pattern Recognition Society of U.S.A. He obtained the 1987 Outstanding Research Award and the 1988-1989, 1990-1991 and 1992-1993 Distinguished Research Awards of the National Science Council of the Republic of China. He also obtained the 1989 Distinguished Teaching Award of the Ministry of Education of the Republic of China. He was the winner of the 1989 , 1992 and 1994 AceR Long Term Award for Outstanding Ph.D. Dissertation and Master Thesis Supervision, the 1991 Xerox Foundation Award for Ph.D. Dissertation Study Supervision, and the 31st S. K. Chuang Foundation Award for Science Research. He was also the winner of the 1990 Outstanding Paper Award of the Computer Society of the Republic of China, and the winner of the 1993 and 1994 Outstanding Paper Award of the Chinese Image Processing and Pattern Recognition Society. Dr Tsai has published more than seventy papers in well-known international journals. Dr Tsai is a senior member of the IEEE, and a member of the Chinese Image Processing and Pattern Recognition Society, the Computing Linguistics Society of the Republic of China, and the Medical Engineering Society of the Republic of China. 